\title{
Plasma natriuretic peptides during supraventricular tachycardia: A study in patients with atrioventricular nodal reentry tachycardia
}

\author{
Redi Pecini $^{1 *}$, Steen Pehrson ${ }^{1}$, Xu Chen ${ }^{1}$, Anna Margrethe Thøgersen ${ }^{2}$, Andreas Kjær ${ }^{3}$, \\ Jesper Hastrup Svendsen ${ }^{1,4,5}$ \\ ${ }^{1}$ Department of Cardiology, The Heart Centre, Rigshospitalet, University of Copenhagen, Copenhagen, Denmark \\ ${ }^{2}$ Department of Cardiology, Center for Cardiovascular Research, Århus University Hospital, Aalborg Hospital, Aalborg, Denmark \\ ${ }^{3}$ Department of Clinical Physiology, Nuclear Medicine and PET \& Cluster for Molecular Imaging, Rigshospitalet, University of Co- \\ penhagen, Copenhagen, Denmark \\ ${ }^{4}$ The Danish National Research Foundation Centre for Cardiac Arrhythmia, Copenhagen, Denmark \\ ${ }^{5}$ Department of Surgery and Medicine, Faculty of Health Sciences, University of Copenhagen, Copenhagen, Denmark \\ Email: ${ }^{*}$ redpecini@gmail.com
}

Received 16 August 2013; revised 15 September 2013; accepted 25 September 2013

Copyright (C) 2013 Redi Pecini et al. This is an open access article distributed under the Creative Commons Attribution License, which permits unrestricted use, distribution, and reproduction in any medium, provided the original work is properly cited.

\begin{abstract}
Aims: To characterize the plasma levels of the atrial natriuretic peptide (ANP) and brain natriuretic peptide (BNP) in patients with atrioventricular nodal reentry tachycardia (AVNRT), we measured the plasma levels of these peptides before and during tachycardia. Methods: We included 10 consecutive patients scheduled for ablation of typical AVNRT without structural heart disease. Catheters were inserted in the femoral artery, femoral vein, and coronary sinus (CS) prior to the ablation procedure. Blood samples were drawn before and after 3 min of tachycardia to measure plasma levels of ANP and BNP. Right atrial pressure (RAP) was measured at baseline. Results: Of the 10 patients, in three patients it was not possible to induce tachycardia leaving a total of 7 patients available for analysis. Mean age of the seven included patients was $40 \pm 12$ years (mean \pm SD), five were female. ANP levels increased significantly during tachycardia in the artery $(p=0.0009)$ and vein $(p=0.003)$, but only borderline in CS $(p=$ 0.09). BNP levels did not change during tachycardia in any location. Conclusion: ANP levels measured in the peripheral circulation increased acutely during tachycardia due to AVNRT. BNP levels did not increase.
\end{abstract}

Keywords: Atrioventricular Nodal Reentry Tachycardia;

${ }^{*}$ Corresponding author.
Atrial Natriuretic Peptide; Brain Natriuretic Peptide

\section{INTRODUCTION}

Brain natriuretic peptide (BNP) and to a lesser extent atrial natriuretic peptide (ANP) have shown to be of value in the diagnosis of heart failure [1,2]. Furthermore, increased levels of BNP have been shown to predict adverse events in patients with heart failure $[3,4]$, coronary heart disease $[5,6]$ and mitral regurgitation [7].

The importance of ANP and BNP in patients with tachycardias is less well known. In patients with atrial fibrillation, levels of BNP are increased [8], and the levels are higher in patients with persistent and permanent AF than those with paroxysmal AF [9]. In addition, one study of patients with AF found higher levels of BNP in the coronary sinus than in the peripheral circulation. The studies of BNP in patients with supraventricular tachycardias are of limited number $[10,11]$. The measurement of BNP levels in these studies was only done from peripheral blood.

As such, the levels of ANP and BNP in patients with supraventricular arrhythmias have not been well characterized. For this reason, we undertook the present study in patients with atrioventricular nodal reentry tachycardia (AVNRT) without concomitant structural heart disease and without the influence of pre-study medication. We measured ANP and BNP levels directly at the coronary sinus, as well as from arterial and venous peripheral blood with the purpose of evaluating the possible cardiac release. 


\section{METHODS}

\subsection{Study Population}

We included consecutive patients referred to our center for ablation of suspected AVNRT. The patients did not have any other concurrent diseases and did not take any medications. They had not had symptoms of arrhythmia for at least one week prior to the ablation procedure. We performed an echocardiography in each patient the day before the ablation procedure. All patients had normal left ventricular ejection fraction (LVEF) and no signs of structural heart disease. Creatinine levels were also normal.

The study was carried out in accordance to the declaration of Helsinki and was approved by the local ethical committee for Copenhagen, study number KF01-046/04. All patients gave written informed consent.

\subsection{Ablation Procedure}

All antiarrhythmic drugs were withdrawn at least five plasma half-lives prior to the procedure. Two 7 French sheaths were inserted; one in each femoral vein, and a 5 French sheath was positioned in the femoral artery. An additional sheath was also inserted in the coronary sinus. The femoral vein sheaths were used for catheter insertion and for the blood samples. The femoral artery and the coronary sinus sheaths were only used for the purpose of blood sampling.

After insertion of the sheaths and positioning of catheters, the right atrial pressure (RAP) measured and then the first series of blood samples was performed. Blood was drawn sequentially in the same order in each patient, first from the coronary sinus (CS), then from the femoral artery and then from the femoral vein. Sampling from each location took approximately 30 seconds. After the first blood sampling (baseline), programmed electrical stimulation was used to induce the arrhythmia. If the first electrical programmed stimulation was unsuccessful, a second programmed stimulation was tried after infusion of isoprotenerol to achieve an increase in heart rate to about 100 beats per minute. Once the tachycardia occurred, it was allowed to continue for at least three minutes and then the blood sampling was repeated. Thereafter, the arrhythmia was stopped and the ablation of the slow pathway at the atrioventricular node was performed. The right atrial pressure (RAP) was only measured at the baseline. No complications occurred during or after the procedure.

\subsection{ANP and BNP Analysis}

Ten $\mathrm{ml}$ of blood was drawn from each sheath, before and during tachycardia (a total of $60 \mathrm{ml}$ ) into tubes containing EDTA and $500 \mu \mathrm{l}$ aprotinin (Trasylol® $500 \mathrm{KIU} / \mathrm{ml}$;
Bayer, Leverkusen, Germany) and centrifuged at $2000 \times$ $\mathrm{g}$ for $15 \mathrm{~min}$. Then plasma was transferred to glass tubes and immediately frozen and kept at $-80^{\circ} \mathrm{C}$ until analyzed.

BNP was measured by an automated two-site sandwich immunoassay technique using chemiluminescence (ADVIA Centaur; Siemens Healthcare Diagnostics). The physiologically active C-terminal peptide of BNP was measured (77 - 108). The sensitivity of the assay was 2 $\mathrm{pg} / \mathrm{ml}$, and the intra-assay and interassay coefficients of variation were $1.2 \%$ and $2.3 \%$, respectively.

ANP was measured by an enzyme immunoassay according to the instructions of the manufacturer (Biomedica Medicinprodukte $\mathrm{GmbH}$, Vienna, Austria). The lower detection limit for NT-pro-ANP (1-98) was 0.05 $\mathrm{nmol} / \mathrm{l}$, and the intra-assay and interassay coefficients of variation were $2 \%$ and $4 \%$, respectively.

The plasma values of ANP and BNP at the baseline were compared with the values during tachycardia in each patient.

\subsection{Statistical Analysis}

Continuous variables are presented median values and inter-quartile range, and discrete variables are presented as percent. We tested the ANP and BNP values for normal distribution visually with histogram graphs and with the use of Kolmogorov-Smirnov test for normal distribution. Not all the data showed normal distribution. Therefore, we performed logarithmic transformation of the values, after which all values showed normal distribution using the same statistical tests. We used paired t-test for the comparison of ANP and BNP logarithmic values before and during tachycardia. For the correlation analyses, we used the general linear model. A p-value less than 0.05 was considered to indicate statistical significance.

\section{RESULTS}

We initially included 10 patients in the study. They all had episodes with palpitations and 12-lead ECGs performed during the episodes had shown short RP tachycardia. The arrhythmia could not be induced in three patients. These patients were excluded from the study. In all the inducible patients the induced arrhythmia was the clinically relevant tachycardia. There was no evidence of an accessory pathway between the atria and the ventricles. The ablation of the slow pathway for AVNRT at the atrioventricular node was successful in all the patients and the arrhythmia could not be induced thereafter.

The baseline data of the seven included patients are presented in Table 1. Baseline values of ANP and BNP were slightly higher in the coronary sinus, but they were not significantly different from the values in the femoral artery or vein. ANP levels increased significantly during 
arrhythmia in the peripheral circulation (femoral artery and vein) compared to the baseline values (Figure 1 and Table 2), but not in the coronary sinus (Table 2). BNP levels did not change significantly during arrhythmia compared to the baseline values (Table 2).

There was no correlation between the patients' age and the baseline levels of ANP and BNP, or the levels of these hormones during tachycardia. Similarly, there was no correlation between the patients' age and the changes of the hormones during tachycardia. Neither did we find a correlation between the ANP and BNP levels at baseline, during tachycardia, or their changes, and RAP.

\section{DISCUSSION}

We measured changes of ANP and BNP during tachycardia in a small group of patients with AVNRT. Our data show that, in the peripheral circulation, ANP levels rose significantly in response to tachycardia. In constrast, BNP levels did not change significantly. ANP is predominately found in the atrial tissue $[12,13]$, while BNP is predominantly found in the ventricles [13]. Both hor-

Table 1. Baseline data of the patients.

\begin{tabular}{cc}
\hline Variable & Value \\
\hline Age (years) & $40 \pm 12($ mean \pm SD) \\
Female sex, number (percentage) & $5(71)$ \\
Tachycardia cyclus (ms) & $336 \pm 28($ mean \pm SD) \\
RAP (mmHg) & $7 \pm 2($ mean \pm SD) \\
log_ANP, femoral artery & $0.25 \pm 0.16($ mean \pm SD) \\
log_ANP, femoral vein & $0.25 \pm 0.19($ mean \pm SD) \\
log_ANP, coronary sinus & $0.34 \pm 0.21($ mean \pm SD) \\
log_BNP, femoral artery & $1.49 \pm 0.43($ mean \pm SD) \\
log_BNP, femoral vein & $1.34 \pm 0.43($ mean \pm SD) \\
log_BNP, coronary sinus & $1.67 \pm 0.39($ mean \pm SD) \\
\hline
\end{tabular}

RAP: right atrial pressure. mones are released into the circulation in response to volume overload and stretch mechanisms [14,15]. In experimental studies, which simulate ventricular overloading conditions, BNP production and release is stimulated more quickly than that of ANP [16]. Our results show the contrary. Two factors may have contributed to our results. First, we did not apply volume-overloading conditions in our patients. It is possible that the stretch stimulus during tachycardia was higher in the thin-walled atria than in the thick-walled ventricles. Second, our patients had no evidence of structural heart disease. BNP baseline levels rise in different pathological conditions of the heart and this increase has prognostic value [3-7]. The failure of BNP to rise during tachycardia in our patients may in part explain the good prognosis in patients with AVNRT and no structural heart disease.

Another finding of our study was that the ANP levels increased in the peripheral sites (femoral artery and vein) but not in the coronary sinus. This finding is somehow surprising. The particular anatomy of the left atrium could be an explanation for this finding. The veins, which drain the posterosuperior part of left atrium, empty directly into the left atrium itself, and not into the coro-

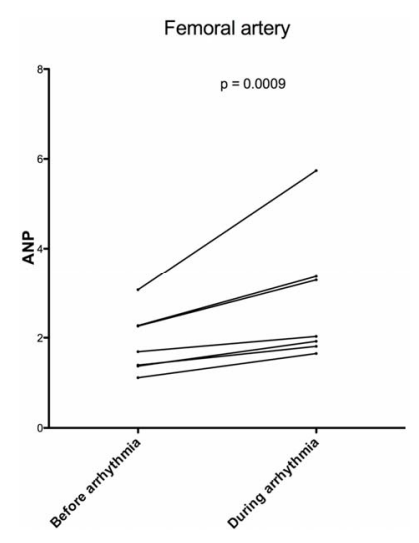

(a)

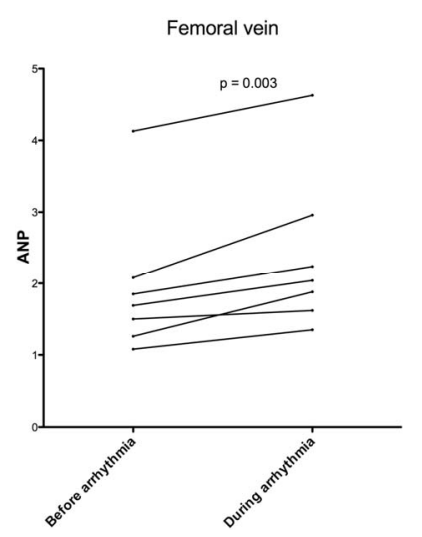

(b)
Figure 1. Changes of ANP levels during tachycardia measured in the femoral artery (a) and changes of ANP levels during tachycardia measured in the femoral vein (b).

Table 2. ANP and BNP changes during tachycardia.

\begin{tabular}{|c|c|c|c|c|}
\hline Variable & Baseline & During tachycardia & Change & p-value \\
\hline log_ANP femoral artery & $0.25 \pm 0.16($ mean $\pm \mathrm{SD})$ & $0.42 \pm 0.21($ mean $\pm \mathrm{SD})$ & $0.17 \pm 0.04$ & 0.0009 \\
\hline log_ANP femoral vein & $0.25 \pm 0.19($ mean $\pm \mathrm{SD})$ & $0.34 \pm 0.18($ mean $\pm \mathrm{SD})$ & $0.10 \pm 0.05($ mean $\pm \mathrm{SD})$ & 0.003 \\
\hline log_ANP coronary sinus & $0.34 \pm 0.21($ mean $\pm \mathrm{SD})$ & $0.50 \pm 0.22($ mean $\pm \mathrm{SD})$ & $0.16 \pm 0.20($ mean $\pm \mathrm{SD})$ & 0.1 \\
\hline log_BNP femoral artery & $1.49 \pm 0.43($ mean $\pm \mathrm{SD})$ & $1.60 \pm 0.37($ mean $\pm S D)$ & $0.12 \pm 0.15($ mean $\pm \mathrm{SD})$ & 0.1 \\
\hline log_BNP femoral vein & $1.34 \pm 0.43($ mean $\pm \mathrm{SD})$ & $1.43 \pm 0.36($ mean $\pm \mathrm{SD})$ & $0.09 \pm 0.13($ mean $\pm \mathrm{SD})$ & 0.1 \\
\hline $\log$ BNP coronary sinus & $1.67 \pm 0.39($ mean $\pm \mathrm{SD})$ & $1.64 \pm 0.41($ mean $\pm \mathrm{SD})$ & $-0.03 \pm 0.11($ mean $\pm \mathrm{SD})$ & 0.6 \\
\hline
\end{tabular}

ANP: atrial natriuretic peptide. BNP: B-type natriuretic peptide. 
nary sinus. This amount of blood empties then in the left ventricle. Thus, the majority of this amount of blood circulates directly into the periphery, and only a minor amount into the coronary artery system. One other explanation could be our sequential method of blood sampling, starting in the coronary sinus. In any case, there is little doubt that the increased levels of ANP are directly related to the tachycardia episode.

Even in patients with lone atrial fibrillation, baseline BNP levels are increased [8], and these levels are even higher in patients with persistent and permanent atrial fibrillation $[9,17,18]$. On the other hand, atrial fibrillation is an arrhythmia, which is associated with considerable morbidity and mortality [19]. On the contrary, AVNRT is not associated with increased mortality and the treatment is usually guided by the burden of symptoms. Furthermore, recent data have shown that the BNP levels in patients with atrial fibrillation are especially increased in the coronary sinus [20]. We did not detect any increase of BNP in blood samples from coronary sinus. This is another fundamental difference between these two arrhythmias. Whether this difference in BNP levels can explain the difference in the morbidity between atrial fibrillation and AVNRT is unsure. However, we know that in other cardiac pathologies, morbidity and mortality risk is closely related to the levels of BNP [4-7].

\section{LIMITATIONS}

Our number of patients was limited. For this reason, we may have overlooked changes in BNP levels due to inadequate power. It is also possible that if we had repeated the measurements of BNP later on we might have been able to detect a change in the BNP levels.

\section{CONCLUSION}

Peripheral plasma levels of ANP but not BNP rose acutely in response to tachycardia in patients with AVNRT. This finding is in contrast to patients with chronic atrial fibrillation indicating the different pathophysiology involved in the two conditions.

\section{ACKNOWLEDGMENTS}

Special thanks to Jens Peter Goetze MD, for his valuable comments on the manuscript.

\section{REFERENCES}

[1] Maisel, A.S., Krishnaswamy, P., Nowak, R.M., et al. (2002) Rapid measurement of B-type natriuretic peptide in the emergency diagnosis of heart failure. New England Journal of Medicine, 347, 161-167. http://dx.doi.org/10.1056/NEJMoa020233

[2] McDonagh, T.A., Robb, S.D., Murdoch, D.R., et al.
(1998) Biochemical detection of left-ventricular systolic dysfunction. Lancet, 351, 9-13.

http://dx.doi.org/10.1016/S0140-6736(97)03034-1

[3] Berger, R., Huelsman, M., Strecker, K., et al. (2002) Btype natriuretic peptide predicts sudden death in patients with chronic heart failure. Circulation, 105, 2392-2397. http://dx.doi.org/10.1161/01.CIR.0000016642.15031.34

[4] Anand, I.S., Fisher, L.D., Chiang, Y.T., et al. (2003) Changes in brain natriuretic peptide and norepinephrine over time and mortality and morbidity in the Valsartan Heart Failure Trial (Val-HeFT). Circulation, 107, 12781283.

http://dx.doi.org/10.1161/01.CIR.0000054164.99881.00

[5] de Lemos, J.A., Morrow, D.A., Bentley, J.H., et al. (2001) The prognostic value of B-type natriuretic peptide in patients with acute coronary syndromes. New England Journal of Medicine, 345, 1014-1021. http://dx.doi.org/10.1056/NEJMoa011053

[6] Schnabel, R., Lubos, E., Rupprecht, H.J., et al. (2006) B-type natriuretic peptide and the risk of cardiovascular events and death in patients with stable angina: Results from the AtheroGene study. Journal of the American College of Cardiology, 47, 552-558. http://dx.doi.org/10.1016/i.jacc.2005.09.039

[7] Detaint, D., Messika-Zeitoun, D., Avierinos, J.F., et al. (2005) B-type natriuretic peptide in organic mitral regurgitation: Determinants and impact on outcome. Circulation, 111, 2391-2397. http://dx.doi.org/10.1161/01.CIR.0000164269.80908.9D

[8] Li, J. and Wang, L. (2006) B-type natriuretic peptide levels in patients with paroxysmal lone atrial fibrillation. Heart Vessels, 21, 137-140. http://dx.doi.org/10.1007/s00380-005-0884-y

[9] Yamada, T., Murakami, Y., Okada, T., et al. (2007) Plasma brain natriuretic peptide level after radiofrequency catheter ablation of paroxysmal, persistent, and permanent atrial fibrillation. Europace, 9, 770-774. http://dx.doi.org/10.1093/europace/eum157

[10] Chen, L., Wei, T., Zeng, C., et al. (2005) Effect of radiofrequency catheter ablation on plasma B-type natriuretic peptide. Pacing and Clinical Electrophysiology, 28, 200-204.

http://dx.doi.org/10.1111/j.1540-8159.2005.09386.x

[11] Cakmak, N., Sayar, N., Cakmak, M., et al. (2008) Effects of radiofrequency catheter ablation on myocardial performance index and plasma NT-Pro-BNP levels in patients with Wolff-Parkinson-White syndrome. Echocardiography, 25, 692-698. http://dx.doi.org/10.1111/j.1540-8175.2008.00669.x

[12] Kangawa, K. and Matsuo, H. (1984) Purification and complete amino acid sequence of alpha-human atrial natriuretic polypeptide (alpha-hANP). Biochemical and Biophysical Research Communications, 118, 131-139. http://dx.doi.org/10.1016/0006-291X(84)91077-5

[13] Ogawa, Y., Nakao, K., Mukoyama, M., et al. (1991) Natriuretic peptides as cardiac hormones in normotensive and spontaneously hypertensive rats. The ventricle is a major site of synthesis and secretion of brain natriuretic peptide. Circulation Research, 69, 491-500. 
http://dx.doi.org/10.1161/01.RES.69.2.491

[14] de Zeeuw, D., Janssen, W.M. and de Jong, P.E. (1992) Atrial natriuretic factor: Its (patho)physiological significance in humans. Kidney International, 41, 1115-1133. http://dx.doi.org/10.1038/ki.1992.172

[15] Yoshimura, M., Yasue, H., Okumura, K., et al. (1993) Different secretion patterns of atrial natriuretic peptide and brain natriuretic peptide in patients with congestive heart failure. Circulation, 87, 464-469. http://dx.doi.org/10.1161/01.CIR.87.2.464

[16] Nakagawa, O., Ogawa, Y., Itoh, H., et al. (1995) Rapid transcriptional activation and early mRNA turnover of brain natriuretic peptide in cardiocyte hypertrophy. Evidence for brain natriuretic peptide as an "emergency" cardiac hormone against ventricular overload. Kidney International, 96, 1280-1287. http://dx.doi.org/10.1172/JCI118162

[17] Therkelsen, S.K., Groenning, B.A., Kjaer, A., et al. (2008) ANP and BNP in atrial fibrillation before and after cardioversion and their relationship to cardiac volume and function. International Journal of Cardiology, 127, 396399. http://dx.doi.org/10.1016/j.ijcard.2007.04.059

[18] Nilsson, B., Goetze, J.P., Chen, X., et al. (2009) Increased NT-pro-B-type natriuretic peptide independently predicts outcome following catheter ablation of atrial fibrillation. Scandinavian Journal of Clinical \& Laboratory Investigation, 69, 843-850. http://dx.doi.org/10.3109/00365510903318209

[19] Camm, A.J., Kirchhof, P., Lip, G.Y., et al. (2010) Guidelines for the management of atrial fibrillation: The Task Force for the Management of Atrial Fibrillation of the European Society of Cardiology (ESC). Europace, 12, 1360-1420. http://dx.doi.org/10.1093/europace/euq350

[20] Gould, P.A., Gula, L.J., Bhayana, V., et al. (2010) Characterization of cardiac brain natriuretic peptide release in patients with paroxysmal atrial fibrillation undergoing left atrial ablation. Circulation: Arrhythmia and Electrophysiology, 3, 18-23. http://dx.doi.org/10.1161/CIRCEP.108.831586 